\title{
Identifying new targets for rectal cancer treatment
}

\author{
Sylvain Ferrandon ${ }^{1}$ and Matthew F. Kalady ${ }^{1,2}$ \\ ${ }^{1}$ Cancer Biology Department, Lerner Research Institute, Cleveland Clinic, Cleveland, Ohio, USA \\ 2 Department of Colorectal Surgery, Digestive Disease and Surgery Institute, Cleveland Clinic, Cleveland, Ohio, USA \\ Correspondence to: Matthew F. Kalady, email: kaladym@ccf.org \\ Keywords: COASY; rectal cancer; radiation resistance \\ Received: April 10, 2020 \\ Accepted: April 14, 2020 \\ Published: May 14, 2020 \\ Copyright: ( 2020 Ferrandon and Kalady. This is an open-access article distributed under the terms of the Creative Commons Attribution \\ License 3.0 (CC BY 3.0), which permits unrestricted use, distribution, and reproduction in any medium, provided the original author and \\ source are credited.
}

Colorectal cancer remains one of the highest incident and top causes of cancer-related mortality in the United States and around the world. In addition to oncologic outcomes, rectal cancer in particular presents additional challenges as surgery for rectal cancer can cause significant decreases in bowel function and quality of life, or even result in a permanent colostomy. The adoption of multimodality care with radiation and chemotherapy before surgery has improved cancer outcomes. In fact, response to neoadjuvant chemoradiation is directly correlated with survival [1]. Furthermore, a subset of patients with an excellent clinical response to chemoradiation may avoid surgery and preserve function [2]. Unfortunately, there is great variety among patients in their response to treatment, and radiation resistance remains a major challenge to successful rectal cancer care [3]. Identifying reasons for this heterogeneity in response, and discovering novel targets to improve radiation sensitivity will lead to treatment advances. We have recently discovered Coenzyme A synthase (COASY) as a novel protein that serves both as a predictive marker for, and has a critical function in radiation resistance [4].

To evaluate patient heterogeneity, we analyzed $C O A S Y$ expression in pretreatment human rectal cancers and found levels varied among patients, and that increased levels were directly related to radiation resistance. Using genetic manipulation with COASY knockdown and overexpression lines, this observation was confirmed in in vitro and in vivo models. Mechanistically, we demonstrated a direct interaction between COASY protein and the PI3K regulatory subunit PI3K-P85 $\alpha$, which increased AKT and mTOR phosphorylation, enhancing cell survival after irradiation. Additionally, shRNA COASY-knockdown increased DNA doublestrand breaks after irradiation [4].

COASY was first described as the mitochondrial enzyme that catalyzes the two last steps of Coenzyme A synthesis in humans. The first reaction, mediated by the
PPAT (4'phosphopantetheine adenylyltransferase) domain, is a transfer of the adenosine monophosphate moiety of ATP to 4'-phosphopantetheine to form dephospho-CoA in an $\mathrm{Mg}^{2+}$ dependent manner. The last step in CoA synthesis is catalyzed by the dephospho-CoA kinase (DPCK) domain of COASY that adds a phosphate group from ATP to the 3'-hydroxyl of dephospho-CoA [5]. However, the newly discovered non-canonical functions of COASY have broadened its implications in cell survival. COASY has been implicated in the maintenance of DNA integrity and mitotic fidelity in different models such as zebrafish and human breast cancer cells [6,7]. Recently, a hot spot mutation in the COASY gene was observed in a subset of neurodegeneration patients with brain iron accumulation (NBIA) and a distinct set of symptoms, accordingly named COASY protein-associated neurodegeneration (CoPAN). These non-canonical functions of COASY are suspected to be altered and responsible for the cell death observed in the CoPAN phenotype.

A proline-rich domain has been found in the N-terminal extension of COASY. This 29 amino-acids sequence is responsible for the protein-protein interaction with the SH3 domains of the Src family non-receptor tyrosine kinases, Fyn and Csk [8]. The interaction leads to a global phosphorylation of tyrosine residues present in COASY which is necessary for its interaction with PI3K-P85 $\alpha$ to promote the activation of the PI3K pathway [9]. Moreover, other proteins are known to interact with PI3K-P85 $\alpha$ and exert a cell protection to radiation by modulating the PI3K complex enzymatic activity, such as Fyn, LYN etc. [10,11]. Our identification of COASY as a key mediator of PI3K/Akt radiation resistance signaling provides additional rational for developing COASY protein-targeted therapeutics.

Our findings may have broader implications beyond rectal cancer as the level of $C O A S Y$ was found to be significantly overexpressed in tumors compared to normal tissues in several other cancers [4]. Furthermore, the ubiquitous role of PI3K/Akt signaling in cancer suggests 
that our results may be relevant to several cancer types. This may play a role in other cancers that have variable responses to radiation. By delineating the underlying mechanisms and a link with PI3K pathway activation, our work sheds light on the potential of COASY as a new therapeutic target for future study as a potential radiationsensitizing agent.

\section{CONFLICTS OF INTEREST}

The authors declare no potential conflicts of interest.

\section{REFERENCES}

1. Mace AG, Pai RK, Stocchi L, Kalady MF. American Joint Committee on Cancer and College of American Pathologists regression grade: a new prognostic factor in rectal cancer. Dis Colon Rectum. 2015; 58:32-44. https://doi.org/10.1097/DCR.0000000000000266. PMID:25489692

2. van der Valk MJ, Hilling DE, Bastiaannet E, MeershoekKlein Kranenbarg E, Beets GL, Figueiredo NL, Habr-Gama A, Perez RO, Renehan AG, van de Velde CJ, Ahlberg M, Appelt A, Asoglu O, et al, and IWWD Consortium. Longterm outcomes of clinical complete responders after neoadjuvant treatment for rectal cancer in the International Watch \& Wait Database (IWWD): an international multicentre registry study. Lancet. 2018; 391:253745. $\quad$ https://doi.org/10.1016/S0140-6736(18)31078-X. PMID:29976470

3. Karagkounis G, Thai L, Mace AG, Wiland H, Pai RK, Steele SR, Church JM, Kalady MF. Prognostic Implications of Pathological Response to Neoadjuvant Chemoradiation in Pathologic Stage III Rectal Cancer. Ann Surg. 2019; 269:1117-23. https://doi.org/10.1097/ SLA.0000000000002719. PMID:31082910

4. Ferrandon S, DeVecchio J, Duraes L, Chouhan H, Karagkounis G, Davenport J, Orloff M, Liska D, Kalady MF. CoA Synthase (COASY) Mediates Radiation Resistance via PI3K Signaling in Rectal Cancer. Cancer Res. 2020; 80:334-46. https://doi.org/10.1158/0008-5472. CAN-19-1161. PMID:31704889

5. Leonardi R, Jackowski S. Biosynthesis of Pantothenic Acid and Coenzyme A. Ecosal Plus. 2007; 2. https://doi. org/10.1128/ecosalplus.3.6.3.4. PMID:26443589

6. Bosveld F, Rana A, van der Wouden PE, Lemstra W, Ritsema M, Kampinga HH, Sibon OC. De novo CoA biosynthesis is required to maintain DNA integrity during development of the Drosophila nervous system. Hum Mol Genet. 2008; 17:2058-69. https://doi.org/10.1093/hmg/ ddn105. PMID: 18407920

7. Lin CC, Kitagawa M, Tang X, Hou MH, Wu J, Qu DC, Srinivas V, Liu X, Thompson JW, Mathey-Prevot B, Yao TP, Lee SH, Chi JT. CoA synthase regulates mitotic fidelity via CBP-mediated acetylation. Nat Commun.
2018; 9:1039. https://doi.org/10.1038/s41467-018-034226. PMID:29531224

8. Breus OS, Panasyuk GG, Gout IT, Filonenko VV, Nemazanyy IO. Proline rich regions of coenzyme A synthase $\alpha$ and $\beta$ interact with $\mathrm{SH} 3$ domains of signaling proteins in vitro. Biopolymers Cell. 2008; 24:123-28. https://doi. org/10.7124/bc.000799.

9. Breus O, Panasyuk G, Gout IT, Filonenko V, Nemazanyy I. CoA synthase is in complex with p85alphaPI3K and affects PI3K signaling pathway. Biochem Biophys Res Commun. 2009; 385:581-85. https://doi.org/10.1016/j. bbrc.2009.05.102. PMID:19482007

10. Saksena S, Gill RK, Tyagi S, Alrefai WA, Ramaswamy K, Dudeja PK. Role of Fyn and PI3K in H2O2-induced inhibition of apical Cl-/OH- exchange activity in human intestinal epithelial cells. Biochem J. 2008; 416:99-108. https://doi.org/10.1042/BJ20070960. PMID:18564062

11. Tornillo G, Knowlson C, Kendrick H, Cooke J, Mirza H, Aurrekoetxea-Rodríguez I, Vivanco MD, Buckley NE, Grigoriadis A, Smalley MJ. Dual Mechanisms of LYN Kinase Dysregulation Drive Aggressive Behavior in Breast Cancer Cells. Cell Rep. 2018; 25:3674-3692.e10. https://doi. org/10.1016/j.celrep.2018.11.103. PMID:30590041 